\title{
Capital social et gestion des conflits dans le périmètre irrigué de Malanville au Bénin
}

\author{
Paul Kouété Jimmy* et Ismail Moumouni Moussa \\ Département d'économie et de sociologie rurales, Faculté d'agronomie, Université de Parakou, BP 1269, Parakou, Bénin
}

\begin{abstract}
Résumé - Cet article analyse l'influence du capital social dans la gestion des conflits au sein des systèmes d'irrigation autogérés à travers le cas du périmètre irrigué de Malanville au Bénin. Il montre que le capital social d'attachement est efficace lorsque le conflit est lié au non-respect des règles opérationnelles. Le capital social de contact contribue à la destruction du capital social d'attachement dans un conflit lié au non-respect des règles collectives et constitutionnelles. La médiation des services de l'État est alors nécessaire pour résoudre le conflit. Ces résultats suggèrent une nécessaire synergie entre les structures autogérées et l'État, pour la résolution effective des conflits.
\end{abstract}

Mots clés : associations d'usagers de l'eau / Bénin / capital social / conflits / périmètre irrigué autogéré

Abstract - Social capital and conflict management in the irrigated scheme of Malanville in Benin. This paper analyses the influence of social capital in conflict management within self-governing irrigation systems through the case study of Malanville irrigated scheme in Benin. It shows that water users valorize efficiently bonding social capital when a conflict relates to operational rules. Linking social capital contributes to destroy bonding social capital in a conflict related to collective and constitutional rules. State agencies mediation was therefore required to resolve the conflict. Results suggest that a synergy approach between self-governing systems and state agencies is necessary for a better conflict management.

Keywords: Benin / conflicts / self-governing irrigated scheme / social capital / water user associations

\section{Introduction}

Au milieu des années 1980, de nombreuses études ont illustré les capacités d'action collective des communautés locales autour des ressources communes (Wade, 1987 ; Ostrom, 1990). Ces études ont permis de nuancer le postulat de la tragédie des communs de Hardin (1968) et ont constitué la base théorique pour promouvoir la dévolution de ces ressources. La forme « ressource autogérée par les communautés » (community-based resource management) est très répandue dans le domaine de l'irrigation. Le principal argument sous-tendant cette politique est que le capital social, vu comme les caractéristiques d'une organisation sociale telles que la confiance, les normes et les réseaux facilitant la coopération, qui existe au sein des communautés, favorise la gestion réussie des ressources communes (Ostrom, 1990 ; Pretty, 2003). Mais ces cas ne sont pas simplement généralisables (Ostrom, 1990 ; Meinzen-Dick et Knox, 2001). Deux formes de gestion peuvent être distinguées :

- des systèmes autogérés (self-governance) sans l'implication de l'État (Ostrom, 1990);

\footnotetext{
$\bar{*}$ Auteur de correspondance : jimmykouetepaul@yahoo.fr
}

- des systèmes cogérés (co-management) où l'État joue encore un grand rôle (Raby, 1991).

La littérature sur la gestion des conflits s'intéresse moins aux systèmes autogérés qu'aux systèmes cogérés, dans lesquels les conflits sont supposés inévitables (Yasmi et al., 2011 ; Ratner et al., 2013). Pour Meinzen-Dick et Knox (2001), les systèmes autogérés sont efficaces pour résoudre les conflits internes. Aussi, très peu d'attention est-elle donnée aux luttes de pouvoir au sein de ces systèmes d'action collective (Ratner et al., 2013). De plus, différentes situations conflictuelles peuvent mobiliser différentes expressions du capital social, favorables ou non à la gestion des conflits (Sanginga et al., 2007).

Cet article vise à analyser l'influence du capital social dans la gestion des conflits au sein des systèmes d'irrigation autogérés à travers le cas de l'Union des groupements de producteurs du périmètre irrigué de Malanville (UGPPM) au Bénin. L'UGPPM est la structure faîtière des Groupements mutualistes de producteurs (GMP), qui sont les associations des usagers du périmètre irrigué de Malanville, le plus grand aménagement hydro-agricole réalisé par l'État au Bénin, et dont la gestion a été transférée aux usagers. Notre projet est, 
d'une part, de préciser la nature des conflits existant dans le périmètre et, d'autre part, d'analyser l'utilisation de différents types de capital social pour la gestion de ces conflits.

\section{Cadre analytique}

Pretty (2003) identifie trois types de capital social, qui orientent la façon dont un groupe peut gérer les ressources communes et résoudre les éventuels conflits. Il s'agit du capital social d'attachement (bonding social capital), du capital social d'accointance (bridging social capital), et du capital social de contact (linking social capital). Le capital social d'attachement décrit les liens forts entre les personnes formant les membres d'un groupe primaire dont les valeurs sont partagées (famille, groupe ethnique ou religieux). À l'UGPPM, le capital social d'attachement fait référence aux structures de liens sociaux tels que l'appartenance des coopérateurs aux mêmes groupes ethniques et religieux, à l'échelle des GMP et à celle de l'UGPPM. Le capital social d'accointance décrit les rapports de collaboration avec des groupes externes ou des fédérations faîtières, impliquant des mécanismes de partage d'information et de résolution de conflits (Meinzen-Dick et Knox, 2001). L'UGPPM n'entretient pas de rapports externes pouvant être décrits comme du capital social d'accointance. L'échelle des relations entre les GMP et l'UGPPM en tant que leur organisation faîtière relève du capital social d'attachement, étant donné qu'il s'agit de la même unité organisationnelle. Le capital social de contact décrit l'habileté de la communauté locale à s'engager avec des structures ou personnes externes en position de pouvoir et d'influence telles que des leaders politiques et de riches personnalités (Dahal et Adhikari, 2008). Le capital social de contact de l'UGPPM regroupe les politiciens ou les responsables de sociétés publiques ou privées natifs de la localité, qui interagissent à titre personnel avec les coopérateurs ou groupes de coopérateurs dans le périmètre irrigué, en matière d'enjeux ou de conflits.

Le terme de « conflits » désigne ici toutes les manifestations de différence ou d'incompatibilité d'intérêts et de buts entre les usagers vis-à-vis des règles existantes, que ce soient des disputes non violentes ou des conflits violents persistants (Ratner et al., 2013). Concernant les règles, Ostrom (1992) en distingue trois niveaux, notamment les règles opérationnelles, les règles collectives, et les règles constitutionnelles. Les règles opérationnelles régissent les décisions quotidiennes du périmètre tandis que les règles collectives et constitutionnelles déterminent la définition des organes de décision et leurs pratiques. En matière de règles opérationnelles, les conflits sont associés aux questions directes de droits et de devoirs dans l'utilisation et le maintien des systèmes d'irrigation, notamment le paiement des redevances, la participation aux entretiens collectifs, et l'utilisation de l'eau. En ce qui concerne les règles collectives et constitutionnelles, les conflits concernent les irrégularités dans le fonctionnement des organes décisionnels instaurés au sein de l'UGPPM tels que l'Assemblée générale (AG) ou le Conseil d'administration (CA). Les conflits sont distingués ici comme provenant, d'une part, du non-respect des règles opérationnelles ou, d'autre part, du nonrespect des règles collectives et constitutionnelles. Selon
Sanginga et al. (2007), l'analyse des conflits part de leur description, c'est-à-dire de l'identification de l'objet, des parties prenantes, des limites et interrelations, des origines, des niveaux et des manifestations. En termes de résolution des conflits, six mécanismes généraux existent : la négociation, la médiation, l'arbitrage, l'adjudication, la coercition et l'évitement (Yasmi et al., 2011). La négociation et l'évitement, du fait qu'ils conduisent à la résolution interne du conflit, amoindrissant ses coûts (Ostrom, 1992), relèvent de l'efficacité du capital social. Par contre, la médiation, l'arbitrage, l'adjudication et la coercition, résultant de l'envenimement et de l'éclatement du conflit, traduisent les limites des mécanismes du capital social (Sanginga et al., 2007). L'intervention de l'État (membres du gouvernement et des structures déconcentrées) dans le périmètre pour la médiation répond à son rôle de régulation et de fourniture de services techniques reconnus dans la gestion des systèmes autogérés d'irrigation (MeinzenDick et Knox, 2001). Aussi, cette intervention (extérieure) n'est-elle pas considérée comme faisant partie du capital social de contact.

L'influence des différents types de capital social de l'UGPPM dans l'évolution ou la résolution des conflits sera explorée ici.

\section{Collecte des données et méthode d'analyse}

La collecte des données a été effectuée d'août 2012 à septembre 2012 à travers des entretiens semi-directifs et des focus-groups. Cette période correspondait à une phase active de la deuxième saison de la campagne agricole. Les coopérateurs étaient réguliers dans le périmètre. Cela a favorisé leurs rencontres, individuellement ou en groupe. Nous avons conduit des entretiens semi-directifs avec différents acteurs impliqués dans les conflits. Au total, 25 personnes ont été sélectionnées pour les entretiens semi-directifs en raison de leur implication dans les conflits, comme protagonistes ou comme acteurs de leur résolution. Il s'agit de 16 coopérateurs simples, de sept responsables de GMP, du technicien agricole spécialisé en inspection et éducation coopératives de Malanville, et le directeur à l'information, à la formation, et à l'appui aux organisations paysannes de la région. En outre, deux focusgroups regroupant des coopérateurs ont permis une triangulation des informations collectées lors des discussions individuelles.

En s'inspirant de Sanginga et al. (2007) et Yasmi et al. (2011), les entretiens ont porté sur les causes des disputes entre coopérateurs, l'évolution des conflits et les acteurs internes et externes impliqués, l'impact des conflits sur les actions collectives, les efforts de résolution des conflits et les acteurs externes impliqués.

L'ensemble des entretiens semi-directifs et des focusgroups ont été enregistrés et transcrits. Nous avons alors recherché et analysé :

- Quels conflits émergent et comment ?

- Comment le capital social est mis en œuvre dans la résolution des conflits ?

L'analyse de discours a été employée et les idées les plus expressives des interviewés sont rapportées. 


\section{Conflits liés au non-respect des règles opérationnelles}

\subsection{Le non-paiement des redevances}

Il y a conflit lié au non-paiement des redevances lorsque le CA et le coopérateur ne s'accordent pas sur l'appréciation faite de l'état des parcelles et les redevances demandées. Le paiement des redevances se fait en nature et est fixé à trois sacs de $82 \mathrm{~kg}$ de riz paddy par parcelle de $0,25 \mathrm{ha}$. Mais, la procédure de recouvrement adoptée dans le périmètre donne des possibilités de paiement partiel, en se basant sur l'état normal ou non des parcelles, enregistré au moment de la supervision. La flexibilité de la règle de paiement liée à cette approche conduit à des incompréhensions et des oppositions du fait des suspicions de subjectivité des redevances demandées. Un responsable de CA nous expliquait un cas vécu dans lequel "un coopérateur a son champ normal lorsqu'on a effectué le contrôle. Mais, quand on lui a demandé de payer la totalité de ses redevances, il jugeait que notre appréciation était injuste et souhaitait qu'on lui fasse des réductions... Ce n'était pas notre avis. On ne s'est pas entendu vraiment... ».

Près de $80 \%$ des coopérateurs interrogés ont été impliqués au moins une fois dans ce type de conflit, mais il s'agit simplement des disputes verbales et non violentes.

\subsection{La non-participation aux travaux collectifs d'entretien}

L'entretien ordinaire des systèmes d'irrigation est assuré par les travaux collectifs des coopérateurs sur la base de la programmation des responsables des GMP. Le chargé de l'information appelle par téléphone et se rend même dans les maisons pour diffuser le programme retenu. La présence de tous les coopérateurs est exigée; en cas d'absence, le coopérateur paie 1000 FCFA $(1,5 €)$ d'amende. Souvent, des coopérateurs s'absentent des travaux collectifs d'entretien et refusent de payer l'amende. Ce refus génère des disputes entre les responsables de GMP et le coopérateur absent. Plus de $90 \%$ des coopérateurs enquêtés ont été concernés au moins une fois par ce type de conflit, mais les disputes sont simplement verbales.

\subsection{L'utilisation frauduleuse de l'eau}

Le conflit naît de l'utilisation frauduleuse de l'eau lorsque les aiguadiers - qui sont des membres du CA désignés pour assurer l'ouverture des vannes des canaux secondaires et des tertiaires - détournent l'eau du canal principal ou des canaux secondaires pour desservir un autre quartier hydraulique, qui n'est pas programmé. Un coopérateur nous déclarait que "des gens corrompent les aiguadiers qui, au lieu de commencer l'irrigation par les quartiers hydrauliques programmés, détournent l'eau vers d'autres. Quand les coopérateurs défavorisés le constatent, cela amène des mésententes... ».

Ces conflits n'ont pas engendré de violence dans le périmètre, et seuls environ $20 \%$ des coopérateurs interrogés.

\section{Conflits liés au non-respect des règles collectives et constitutionnelles}

Les textes de l'UGPPM prévoient pour le CA un mandat de trois ans renouvelable une fois. En pratique, l'UGPPM a connu trois phases :

- une première phase, de 1989 à 2001, pendant laquelle les règles ont été respectées, avec deux CA qui se sont succédé, un premier CA ayant fait deux mandats, puis un second ayant fait également deux mandats ;

- une deuxième phase, de 2001 à 2011, pendant laquelle le CA élu n'a pas respecté les règles et est resté en place pendant dix ans, ce qui a engendré un conflit ;

- une troisième phase, depuis 2011, de retour au respect des règles avec l'installation d'un nouveau bureau après la résolution du conflit.

\subsection{Le non-respect des principes démocratiques}

Le premier président du CA (1989-1995) a été rappelé à la tête de l'organisation en 2001, avec son bureau. Ils sont restés en place pendant près de dix ans, en violation des textes qui établissent un mandat de trois ans, renouvelable une seule fois. De plus, durant cette période, le bureau n'a pas organisé régulièrement les $\mathrm{AG}$ ordinaires annuelles. Ces $\mathrm{AG}$, qui permettent le respect du principe de redevabilité (accountability) aux membres, n'ont été tenues que les trois premières années sur les dix ans qu'a duré ce « mandat » (qui n'en était plus un puisque le bureau n'était plus mandaté par l'AG), générant une atmosphère de soupçons de mauvaise gouvernance financière. Ce non-respect des règles a conduit le périmètre dans un conflit qui a opposé deux groupes de coopérateurs : le premier groupe correspondant au bureau en place, avec à sa tête le premier président de l'UGPPM (1989-1995) et ses partisans, et le second groupe composé du deuxième président du CA (1995-2011) et ses partisans. Les membres des deux groupes provenaient indistinctement de tous les GMP. De fait, deux CA se sont instaurés dans le périmètre, car le second leader a formé un CA parallèle à celui considéré comme illégitime, pour récupérer les redevances auprès des coopérateurs de son camp. Ce conflit portait sur le rétablissement de l'ordre réglementaire. Un coopérateur nous rapportait que " personne ne doit être irremplaçable dans le périmètre, c'est pourquoi nous voulions qu'il y ait une assemblée générale pour procéder au renouvellement $d u$ bureau du $C A$ ».

Ce conflit révèle que le capital social d'attachement de l'UGPPM a été fragilisé parce que les responsables locaux n'ont pas su se soumettre aux principes démocratiques ni se les approprier.

\subsection{Les relations du conflit avec l'environnement politique extérieur}

La lutte de pouvoir qui a opposé deux groupes de coopérateurs ayant à leur tête les deux premiers présidents de l'UGPPM a trouvé un ancrage politique dans l'environnement extérieur. Un membre du bureau «illégitime » en place interprétant la position de son camp nous expliquait 
qu' " il y avait une main politique derrière la réaction des opposants... Le meneur de jeu dans la crise comptait sur le soutien d'un cadre et leader politique natif de la localité - alors ministre du Gouvernement (il intervenait à titre personnel), dont il a utilisé les promesses de faire bénéficier des projets au périmètre pour drainer d'autres coopérateurs dans son camp ». Les membres de la partie opposante affirmaient que " le directeur général de la SONAPRA (société distributrice d'intrants), cadre et leader politique de notre localité, soutient le bureau du premier président de l'UGPPM... Comme "ils" (les membres du bureau mis en cause) sont du même bord politique que le directeur, ils comptaient s'appuyer sur lui pour garder le $C A »$. De ces deux propos, il ressort que les deux parties prenantes dans ce conflit incriminent les soutiens de leaders politiques de la localité pour une partie ou pour l'autre. L'intervention des politiques se justifie par l'intérêt électoral perçu au niveau du périmètre comme l'a déclaré le directeur à l'information, à la formation, et à l'appui aux organisations paysannes de la région : "Le périmètre représente une masse électorale. C'est pourquoi les leaders politiques de la localité s'en sont mêlés ; ils voudraient que leurs partisans soient à la tête pour relayer leur message en temps électoral... ».

Ainsi, la crise politique au sein de l'UGPPM fait apparaître des contacts politiques à l'extérieur qui, pour leur intérêt propre, renforcent chacune des parties prenantes dans sa position intransigeante, favorisant l'envenimement du conflit. Notamment, le technicien agricole spécialisé en inspection et éducation coopératives de Malanville nous a rapporté qu'" avec ces renforcements partisans, les coopérateurs qui avaient toujours travaillé ensemble dans le périmètre ne se parlaient plus...».

\section{Gestion des conflits liés au non-respect des règles opérationnelles}

Les coopérateurs considèrent les conflits liés au non-respect des règles opérationnelles comme « normaux », c'est-à-dire inhérents à l'action collective. Ainsi, ils ont rapporté fréquemment dans leurs propos au cours du focus-group que : "là où plusieurs personnes travaillent ensemble, il y a toujours de petits problèmes. Le refus de paiement des amendes pour non-participation aux travaux collectifs, le refus de paiement des redevances réclamées par les responsables, et l'utilisation frauduleuse de l'eau sont de simples désaccords ». Une telle minimisation de ces conflits évite leur envenimement et favorise la négociation. En effet, $100 \%$ de ces conflits ont été résolus en interne, soit à l'échelle des GMP pour les conflits liés à la non-participation aux travaux collectifs, soit à celle de l'UGPPM pour les conflits liés au non-paiement des redevances ou à l'utilisation frauduleuse de l'eau. La négociation a consisté pour les protagonistes à transformer les échanges de paroles exprimant les désaccords en échanges exprimant des excuses et la compréhension mutuelle, avec la promesse de mieux agir une autre fois. Il ne s'agit pas d'une instance formalisée pour la résolution de conflits, mais de l'utilisation du capital social d'attachement pour favoriser la négociation.

Tous les coopérateurs interrogés ont mentionné les relations d'ordre familial et religieux existant entre eux pour expliquer leur démarche de maîtrise de ces conflits, notamment ce règlement par consensus entre les parties. Un coopérateur nous affirmait qu' "il est inutile de chercher à se mettre dans des problèmes. Nous sommes coopérateurs dans le périmètre, mais nous sommes aussi liés par des liens familiaux et religieux... ".. La résolution en interne de ces conflits dénote l'efficacité du capital social d'attachement. L'UGPPM, avec la maîtrise de ces conflits, agit ainsi en structure cohésive, même si l'influence sur la qualité de la gestion technique durable du périmètre peut poser question, étant donné la tendance des coopérateurs à privilégier le compromis social à la participation réglementaire effective.

\section{Gestion des conflits liés au non-respect des règles collectives et constitutionnelles}

Le conflit lié au non-respect des règles collectives et constitutionnelles que constitue le non-respect de la durée du mandat et du principe de redevabilité, avec ses relations à l'environnement politique extérieur, n'a pu trouver de solution sans la médiation de l'État. En effet, la division au sein du périmètre et le durcissement des positions des parties prenantes n'ont donné aucune possibilité à la négociation pour une résolution interne. Cette situation conflictuelle persistante est parvenue à l'État par le biais des services déconcentrés en charge de l'agriculture dans la région. La médiation de l'État s'est basée sur la mise en place d'un comité préparatoire de l'AG de l'UGPPM devant organiser cette AG destinée à élire un nouveau bureau. Ce comité a été installé lors d'une séance de concertation avec les coopérateurs, à laquelle ont participé deux membres du gouvernement délégués par le président de la République et des responsables des structures agricoles déconcentrées. Les représentants du gouvernement ont expliqué aux parties prenantes l'enjeu national pour la production rizicole, le périmètre étant le plus grand au niveau national, et donc l'urgence de ramener l'ordre. Le directeur à l'information, à la formation et à l'appui aux organisations paysannes de la région a été désigné à la tête dudit comité. La principale activité du comité a consisté à organiser des réunions avec les coopérateurs pour rappeler les textes de l'Union et expliquer les défis, et recenser les coopérateurs appartenant aux différents GMP et à l'UGPPM. La participation des coopérateurs à ces différentes phases, motivée par le processus de retour à l'ordre démocratique qui était enclenché, a favorisé la tenue de l'AG élective. À l'issue de celle-ci, les membres élus provenaient des deux parties prenantes du conflit et l'unicité du CA de l'UGPPM, et donc celle de l'UGPPM ellemême, a été rétablie. Un coopérateur nous a rapporté que : «les textes ont été dits, et l'ordre démocratique est revenu ".

Nous pouvons noter que le processus de médiation de l'État a permis un dénouement heureux du conflit. Notons aussi que le message du président de la République porté par ses ministres à la réunion de mise en place du comité a réduit les ardeurs belliqueuses et aidé le comité à initier ses activités. Une intervention du gouvernement à cette échelle peut être reliée à l'importance socioéconomique du périmètre comme indiqué dans le message du président de la République, mais elle s'explique surtout par le mode de gouvernance du pouvoir en place qui a pris l'habitude de marquer sa présence dans tous les sujets, à tous les niveaux, en vue de montrer sa proximité avec la population, à des fins politiciennes. 


\section{Discussions et conclusion}

Le présent article apporte des éléments de compréhension sur l'influence du capital social dans la gestion des conflits au sein des systèmes d'irrigation autogérés, en l'occurrence l'UGPPM au Bénin. Les coopérateurs de l'UGPPM ont mis en avant leur appartenance aux mêmes groupes ethniques et religieux pour résoudre les conflits liés au non-respect des règles opérationnelles par la négociation, démontrant ainsi l'efficacité du capital social d'attachement (Sanginga et al., 2007). Mais la résolution du conflit lié au non-respect des règles collectives et constitutionnelles a nécessité la médiation des services de l'État. La crise politique au sein de l'UGPPM a fait apparaître deux camps reliés à des contacts politiques à l'extérieur, qui, pour leur propre intérêt, renforcent les clivages. Le capital social d'attachement a été ainsi fragilisé par l'enjeu politique, alors qu'il a servi de base à la résolution facile des conflits liés au non-respect des règles opérationnelles. Cela montre qu'un type de capital social bénéfique pour un but peut ne pas l'être pour un autre (Sandefur et Laumann, 1998). En outre, le capital social de contact ne promeut pas les pratiques démocratiques (Titeca et Vervisch, 2008) ; des contacts politiques ont ici soutenu les responsables de l'UGPPM qui enfreignaient les principes réglementaires de la durée de mandat. Cela a même encouragé l'envenimement du conflit. C'est ainsi que, ici, contrairement aux résultats de Dahal et Adhikari (2008), le capital social de contact a contribué à la destruction du capital social d'attachement.

Ces résultats montrent que :

- l'efficacité du capital social d'attachement à maintenir une communauté cohésive diminue avec l'enjeu politique ;

- le capital social de contact a un effet " fragmentogène" lorsqu'il provient des leaders politiques « intéressés », et renforce les brèches de divergences «politiques » qui pourraient exister dans la structure locale.

Aussi, tous les types de capital social ne sont-ils pas systématiquement positifs (Sandefur et Laumann, 1998). De plus, l'influence du capital social d'attachement dans la résolution des conflits liés au non-respect des règles opérationnelles ne semble pas privilégier la participation réglementaire des coopérateurs pour la maintenance du périmètre. L'intervention de l'État dans le périmètre, même si elle n'est pas dépourvue d'arrière-pensées « politiciennes », suggère une utile « synergie » (Sanginga et al., 2007) entre les structures de gestion des ressources communes, censées être en totale « autogestion », et l'État, pour la résolution effective des conflits. Mais l'appropriation des principes démocratiques est aussi nécessaire au sein de l'UGPPM pour éviter de retomber à l'avenir dans des vides institutionnels, sources de conflit.

\section{Références}

Dahal GR, Adhikari KP. 2008. Bridging, linking and bonding social capital in collective action: the case of Kalahan Forest Reserve in Phillipines. In: CAPRi Working Paper No. 79. Washington D.C.: International Food Policy Research Institute, http://dx.doi.org/ 10.2499/CAPRiWP79.

Hardin G.1968. The tragedy of the commons. Science 162: $1243-1248$.

Meinzen-Dick RS, Knox A.2001. Collective action, property rights, and devolution of natural resources management: a conceptual framework. In: Meinzen-Dick RS, Knox A, Di Gregorio M, eds. Collective action, property rights, and devolution of natural resources management: exchange of knowledge and implications for policy. Feldafing (Germany): Zentralstelle für Ernährung und Landwirtschaft, pp. 40-72.

Ostrom E.1990. Governing the commons. The evolution of institutions for collective action. Cambridge: Cambridge University Press.

Ostrom E.1992. Crafting institutions for self-governing irrigation systems. San Francisco: ICS Press.

Pretty J.2003. Social capital and the collective management of resources. Science 302: 1912-1914.

Raby N.1991. Participatory management in large irrigation systems: issues for consideration. World Dev 19: 1767-1776.

Ratner BD, Meinzen-Dick R, May C, Haglund E.2013. Resource conflict, collective action, and resilience: an analytical framework. Int $J$ Commons 7: 183-208.

Sandefur RL, Laumann EO.1998. A paradigm for social capital. Ration Soc 10: 481-502.

Sanginga PC, Kamugisha RN, Martin AM.2007. The dynamics of social capital and conflict management in multiple resource regimes: a case of the southwestern highlands of Uganda. Ecol Soc 12: 6.

Titeca K, Vervisch T.2008. The dynamics of social capital and community associations in Uganda: linking capital and its consequences. World Dev 36: 2205-2222.

Wade R.1987. The management of common property resources: collective action as an alternative to privatization or state regulation. Camb J Econ 11: 95-106.

Yasmi Y, Kelley L, Enters T. 2011. Forest conflict in Asia and the role of collective action in its management. In: CAPRi Working Paper No. 102. Washington D.C.: International Food Policy Research Institute, http://dx.doi.org/10.2499/CAPRiWP102.

Citation de l'article : Jimmy PK, Moumouni Moussa I. 2016. Capital social et gestion des conflits dans le périmètre irrigué de Malanville au Bénin. Cah. Agric. 25:65003. 